\title{
On a combined measurement technique of PIV and shadowgraph in environmental fluid dynamics
}

\author{
Tao WANG ${ }^{1}$, Zhaohua YIN ${ }^{2}$, Wenrui $\mathrm{HU}^{3}$ \\ National Microgravity Laboratory, Institute of Mechanics, Chinese Academy of Science \\ Beijing 100190, China
}

\begin{abstract}
In this paper, a combined measurement technique of Particle Image Velocimetry (PIV) and shadowgraph is proposed to investigate the environmental fluid dynamics. This measurement system is based on a PIV system, with a backlight added to produce the shadow of dyed water. The images recorded by a CCD camera are then processed for the PIV and shadowgraph data, separately. The experimental results show that this technique is well suitable for the investigation of time-dependent flow field with temperature variation. Fairly good agreements with the experimental and CFD results are achieved.
\end{abstract}

Keywords: Ventilation, PIV, shadowgraph

\section{INTRODUCTION}

Environmental fluid dynamics has been intensively studied recently for its application in the ventilation control of cities, buildings, and some hermetic environments like space capsules. During the future long-term manned space tours, it is necessary to ameliorate the living conditions of Yu-hang-yuan. Environmental fluid dynamics, especially ventilation, is essential in Environment Control and Life Support System (ECLSS), which controls temperature, humidity, and air comfort in space capsule.

The issue of ventilation concerning indoor air quality has been widely investigated. Linden et al. [1] used the LindenLane-Serff-Smeed theory (LLSS) to study environmental fluid dynamics in natural ventilation. They developed a methodology of small-scale modeling experiment by using water as the working fluid, and the buoyancy forces are produced by salinity differences. Flow visualization with dyed water is performed using shadowgraph to get flow patterns and density variations in their work. Employing digital image-processing techniques, Hacker et al. [2] made quantitative measurements of flow velocities and temperature. But with only shadowgraph data available, they could not get the whole velocity field.

Lindken and Merzkirch [3] adopted a combined PIV and shadowgraph technique with single black/white camera to investigate a bubbly two-phase flow problem. The two phases were separated by the shadowgraph and digital masking techniques. Nogueira et al. [4] and Carpintero-Rogero et al. [5] improved this image processing technique to determinate the bubble shape.

Technically, it is easy to get the detailed velocity field for a ventilation problem with the PIV technique. But for the temperature field, the high-resolution measurement is much more difficult. One method to measure the global temperature field is using the thermo-chromic liquid crystals (TLC). When TLC particles are illuminated, they selectively reflect light at a visible wave length characterized by the local temperature. As the colors of white light scattered from the TLC particles are influenced against temperature and scattering angle, each point of the test field has to be calibrated to compensate for the influence of the illuminating light variation tediously [6]. The limitation of TLC method is that crystals can only display various colors for a narrow range of temperature, hence, only a narrow thermal range can be measured in this method. Laser-induced fluorescence (LIF) with temperature-insensitive fluorescence dye can be also used to measure temperature distribution of the whole flow zone. But the disadvantage of the LIF technique

\footnotetext{
${ }^{1}$ wangtao@imech.ac.cn; phone: 86-10-82544214; fax: 86-10-82544096.

${ }^{2}$ zhaohua.yin@imech.ac.cn

${ }^{3}$ wrhu@imech.ac.cn
}

ICEM 2008: International Conference on Experimental Mechanics 2008, edited by Xiaoyuan He, Huimin Xie, Yilan Kang, Proc. of SPIE Vol. 7375, 73751Q · @ 2009 SPIE · CCC code: 0277-786X/09/\$18 · doi: 10.1117/12.839070 
is that the fluorescence dye introduces an error of around $0.2-1.8$ per ${ }^{\circ} \mathrm{C}$ near $20^{\circ} \mathrm{C}$ (depending on the kinds of fluorescence dye used), and the sensitivity of the dye changes with temperature which requires repeated calibration[7].

In this paper, we adopt the same strategy mentioned in Linden et al. [1] to replace the temperature field of air with the concentration distribution of dyed-salted water. The main objective of this study lies in the accurate acquisition of flow velocity and dye-salt concentration field data through the employment of PIV and shadowgraph techniques. Finally, the experimental results are compared with the CFD results.

\section{MEASUREMENT TECHNIQUE}

\subsection{Experimental facility}

In this work, the PIV images are obtained by seeding the liquid with particles, which are illuminated by a double-cavity pulsed Nd:YAG laser with a wavelength of $532 \mathrm{~nm}$. Through a group of lens, the laser beam is transformed into a laser sheet of about $1 \mathrm{~mm}$ thickness in the test section (with a size of $100 \mathrm{~mm} \times 100 \mathrm{~mm}$, Fig.1). A Dantec HiSense CCD camera, with a resolution of $2048 \times 2048$ pixels with 12 bits (The images are recorded with 8 bits, which gives 256 gray levels, although the CCD camera used allows the acquisition with 12 bits and, therefore, 4096 grey levels), is positioned orthogonal to the laser sheet. The particles are made of polystyrene with a mean diameter of $10 \mu \mathrm{m}$.

\section{Backlight Panel}
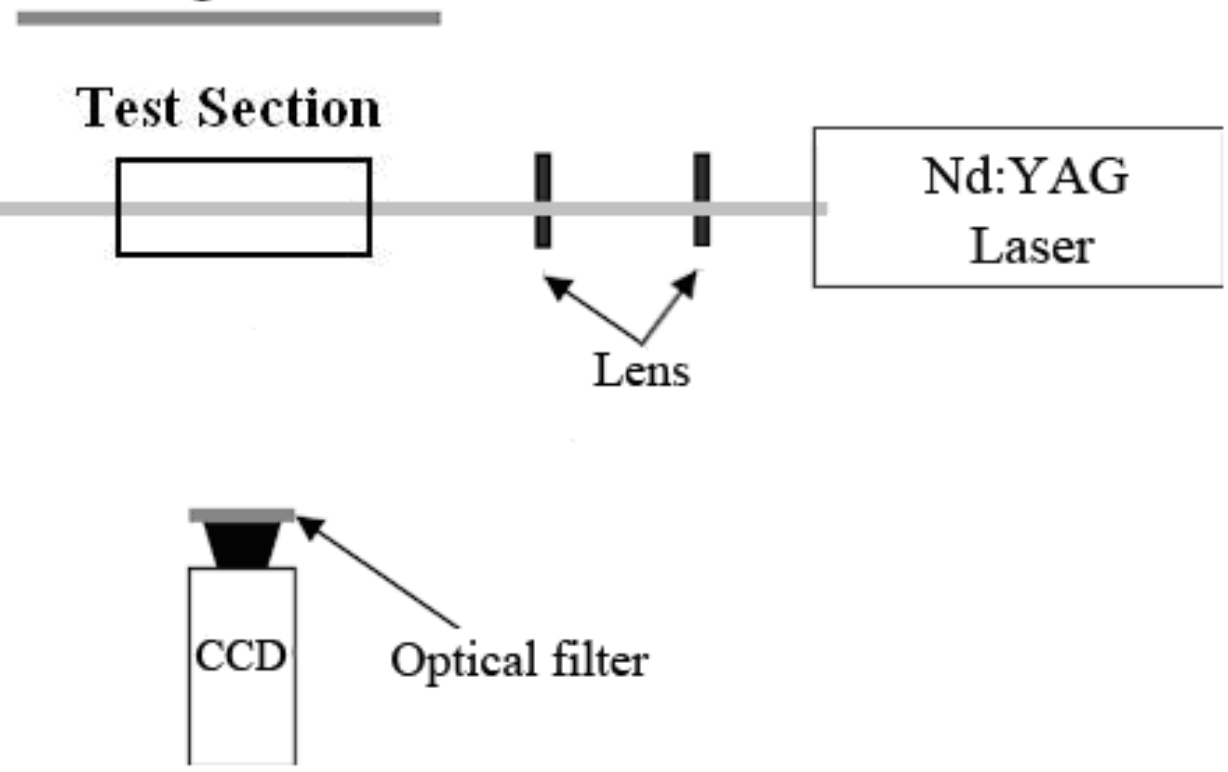

Fig. 1. Sketch of experimental facility

An optical filter is used to block the intense light noises and to allow the passage of the light reflected by particles. A 60$\mathrm{mm}$ focal length lens is adopted to acquire the flow pattern of the test section. The whole PIV System (Dantec Flowmap PIV2100) is controlled by a hardware timing unit. The distance between pulses of cavity 1 and 2 of the Nd:YAG laser is adapted to the respective parameters of the operating point in order to get an optimal particle displacement. The digital camera is focused on the laser light sheet to record the information of the particles of the PIV measurement and the backlight shadow.

To obtain the shadow images, the flow was illuminated by a plane-source backlight. Due to this background illumination, the dyed fluid zones produce a shadow, which is recorded by the CCD camera of the PIV system. Between the backlight panel and the test section, a diffuser plane is added for adjusting the backlight to distinguish the light emitted by the tracer particles from the background lighting. 


\subsection{Image processing}

a) Origin Image

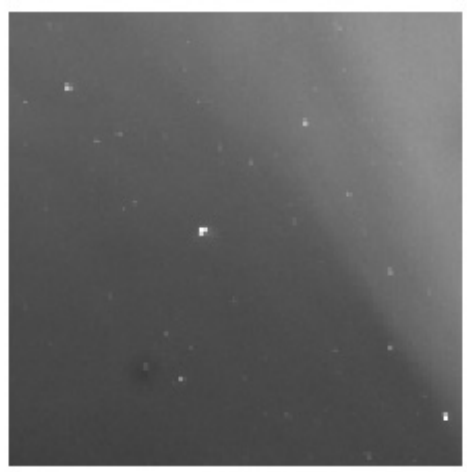

b) Median Filtered [3×3]

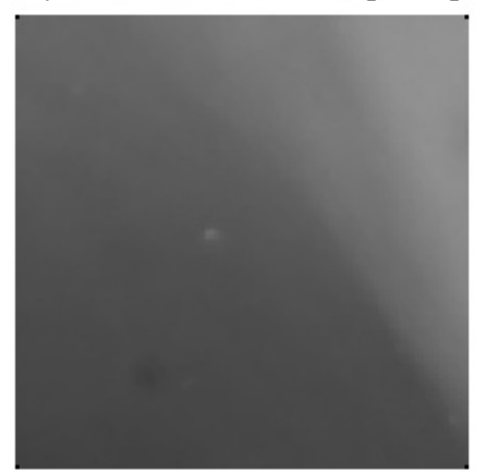

c) Tophat Filtered [3×3]

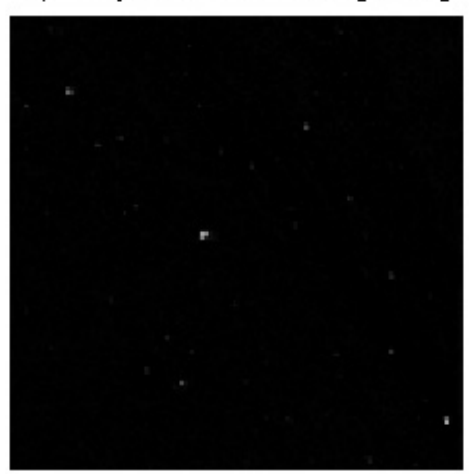

Fig. 2. a) an original experimental image; b) the image processed by the median filter; c) the image processed by the top-hat filter. Both filters are applied on a [3×3] neighborhood.

The original experiment images contain simultaneous information from tracers and dye density. Fig. 2a) shows a typical image got from the experiment. Three kinds of gray levels can be observed:

- The brightest level, which corresponds to the tracer particles;

- The darker part, which is the dye-flushed region (for example, the right-up corner of Fig. 2a);

- The darkest region corresponding to the shadow of the undisturbed dyed fluid zones (for example, the left-bottom corner of Fig. 2a).

To separate the information of tracers and dye density, the experimental images are processed with two kinds of filters:

1) The median filter eliminates the PIV particles to get the shadowgraph images. Each output pixel contains the median value in the given neighborhood around the corresponding pixel in the input image.

2) The top-hat filter eliminates the background color to get the PIV images. There are three steps in this procedure. Firstly, bright areas smaller than the structuring element, which is $3 \times 3$ pixels in our case, are completely removed from the image. Larger bright areas are reduced in size as the surrounding darker pixels flood in from the edges. Secondly, any remaining bright areas are allowed to grow back to their original size by flooding neighboring darker pixels. With all small bright features gone, only the background remains. Finally, the background found is subtracted from the original image, so only bright foreground features smaller than the structuring element remain.

The separation process is carried out with some self-developed Matlab codes.

In practice, there is some subtle difference between the distributions of the dye and those of salt because their diffusion rates are different. In our case, the Peclet number $(\mathrm{Pe}=\mathrm{L} \cdot \mathrm{V} / \mathrm{D}$, the ratio of the convection rate to diffusion rate) is very high $\left(\mathrm{O}\left(10^{4}\right)\right)$, and the diffusion is trivial when compared with the flow convection. Therefore, the diffusivity effect is neglected.

PIV algorithm is directly applied to the top-hat filtered images. The flow field is determined by processing the acquired images with adaptive cross-correlation, moving average and scale filter.

\section{EXPERIMENT}

The experiment set-up is sketched in Fig. 3. Experiments are conducted in a Perspex box (size of $0.1 \mathrm{~m} \times 0.1 \mathrm{~m} \times 0.04 \mathrm{~m}$, referred as the mock-up in the following) containing dyed deionized water. Reservoir 1 containing pure deionized water is placed above the mock-up to create the inlet flow by pressure drop. The inlet flow rate is measured and controlled by an inductive flowmeter before the water enters the mock-up. The outlet flow of the mock-up is reclaimed in the Reservoir 2. Before experiments, PIV tracer particles are added into the water of the mock-up and Reservoir 1 . The 
temperature of the laboratory remains at $25^{\circ} \mathrm{C}$ to keep the whole system thermal isolated. A dark environment was built around the measurement system and the test section in order to avoid disturbing effects of ambient light. During the experiment, the optical filter (Fig. 1) and the roughened bottom plate of mock-up are used to avoid intensive reflections of the laser light sheet.

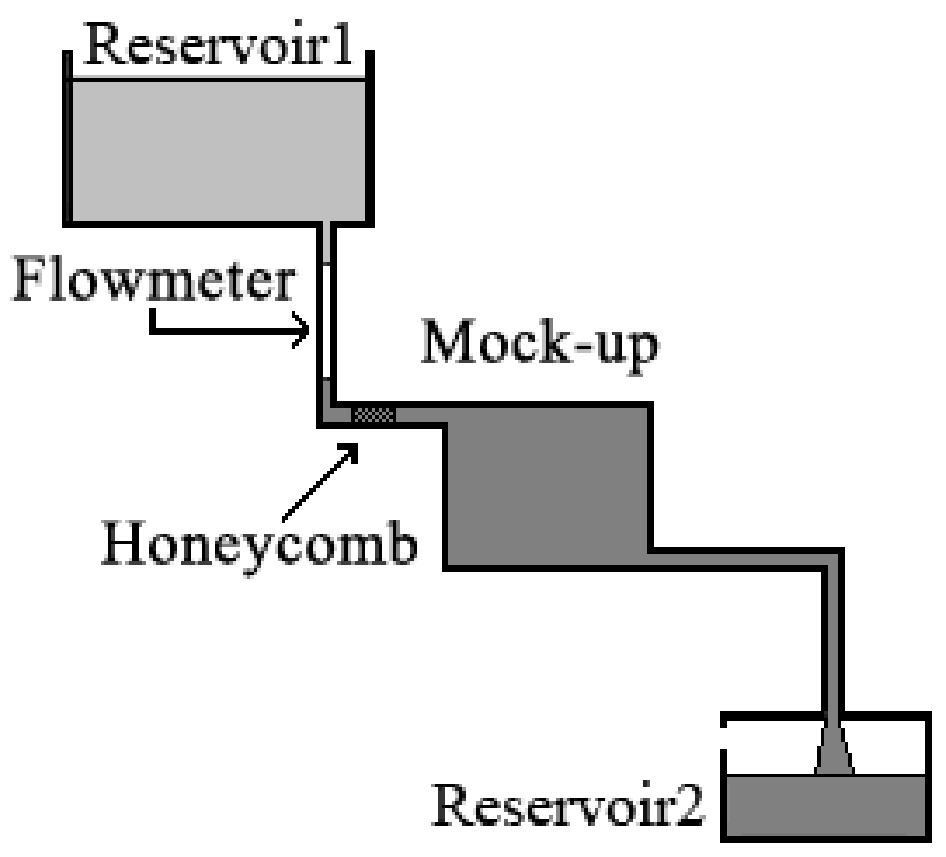

Fig. 3. Experiment set-up

As mentioned above, ventilation flows under gravity are simulated in the laboratory by using brine and fresh water to create density differences. At the beginning of each experiment, the inlet vent is opened, and the pure water in Reservoir 1 enters the mock-up through a honeycomb. The dyed fluid in the mock-up will then be flushed out gradually through the outlet vent. Here, the buoyancy forces act downwards in contrast to the orientation of thermal flow behavior in real ventilation. The brine in the mock-up corresponds to the warm air in the environment, and the whole flow field is simply inverted vertically. This reversal of the direction of the buoyancy force is unimportant to the dynamics, apart from reversing the sense of the motion [8].

\section{RESULTS AND DISCUSSION}

The mock-up is about $1 / 20$ of the targeted space capsule in size. The inlet velocities in experiments vary from $0.083 \mathrm{~m} / \mathrm{s}$ to $0.333 \mathrm{~m} / \mathrm{s}$, corresponding to the air speed range of $0.06 \sim 0.24 \mathrm{~m} / \mathrm{s}$ in the space capsule. The Reynolds number $(\operatorname{Re}=\mathrm{L} \cdot \mathrm{V} / \mathrm{v})$ is $800 \sim 3300$ on the inlet size. A median filter with a neighborhood of $7 \times 7$ pixels has been used to acquire the shadowgraph images, and a tophat filter with a neighborhood $3 \times 3$ pixels for the PIV images. In the PIV algorithm, the initial image has $2048 \times 2048$ pixels, and the initial window size for cross-correlation is $64 \times 64$ pixels. An overlap of $50 \%$ refines the grid spacing to $32 \times 32$ pixels and allows a resolution of $1.56 \mathrm{~mm} \times 1.56 \mathrm{~mm}$ in the measurements. Fig. 4 a)\&c) show the velocity and temperature fields of one typical experimental result. Note that a slab with $1 / 2$ height of the mock-up is placed on the bottom. It appears that the temperature field forms a step-like geometry after the whole system becomes equilibrium. There is almost no dye in the space higher than the slab and on the right of the slab, but the dye on the left corner is nearly undisturbed (Fig. 4c). To save computing time, a two-dimensional (2D) mode is solved in our numerical simulation. Fig. 4b)\&d) are numerical results from the commercial CFD software-FLUENT at the same time as the experimental results in Fig 4a)\&c). Although the actual three-dimensional experiment is simulated by the 2D model, the experiment and CFD results are still in fairly good agreements. 


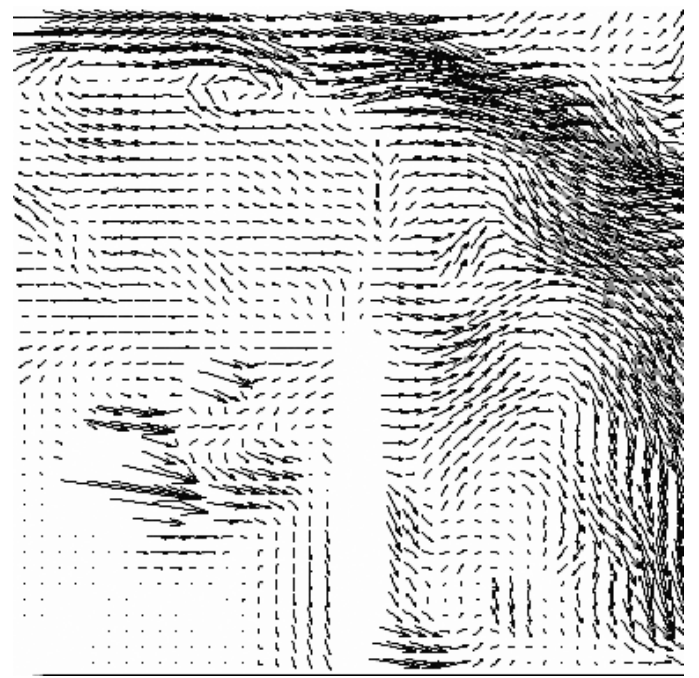

a)

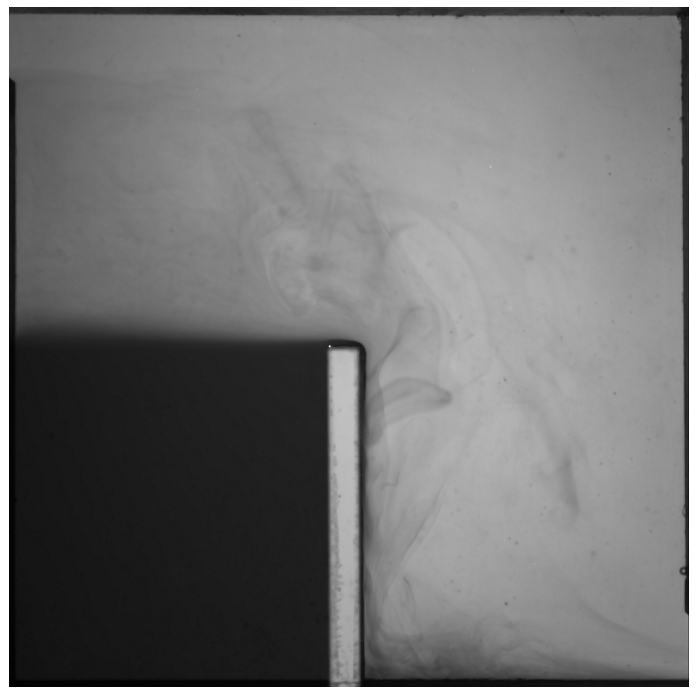

c)

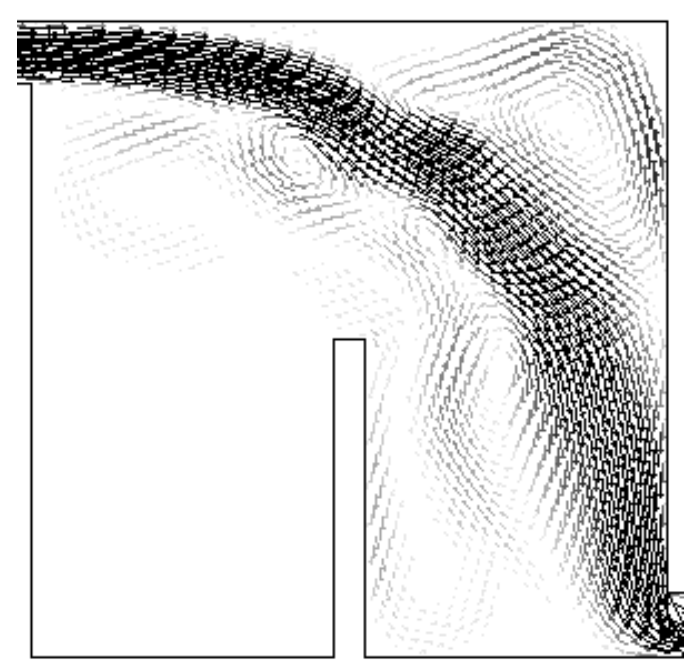

b)

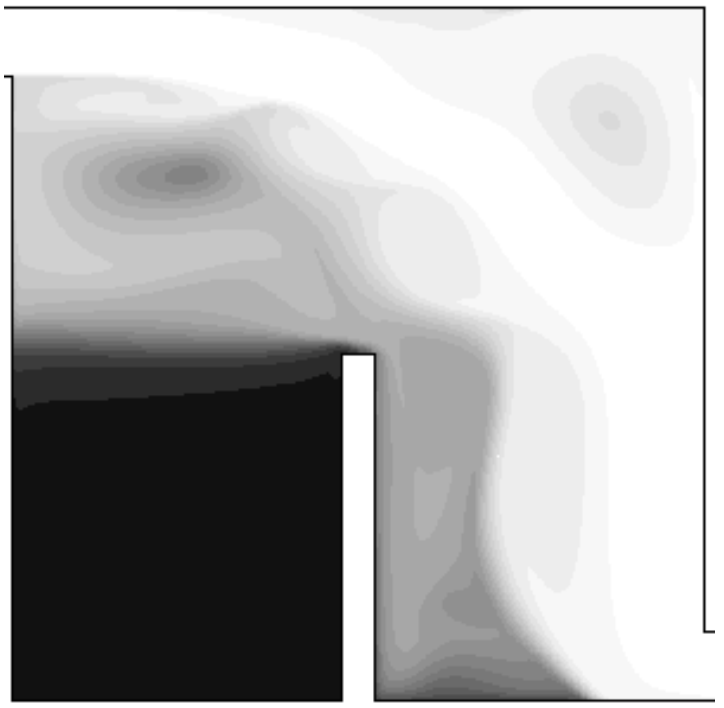

d)

Fig. 4. Comparison of experimental data and CFD results: a) the velocity field from the experiment; b) the velocity field from CFD; c) the shadowgraph picture; d) the temperature contours from CFD. All images are sample at $t=34 \mathrm{~s}$ with an inlet velocity of $0.083 \mathrm{~m} / \mathrm{s}$. The height of inlet vent is $10 \mathrm{~mm}$.

\section{CONCLUSION}

In this work, a combined PIV and shadowgraph technique is adopted to investigate a ventilation system. It seems that the strategy is suitable in the study of the simultaneous information of temperature distribution and flow field. At the beginning of each experiment or in some specific positions of models, when dense dyes fill the flow zone, the reflected light from PIV particles is weak, so the accuracy of PIV procedure may be affected. Thus, it still needs to be work on to improve the accuracy of experimental data in the following researches. In the future, the same method will be used to investigate more complicated cases such as mock-ups with more complicated geometrics or with thermal sources. 


\section{ACKNOWLEDGEMENTS}

This work is supported by National Natural Science Foundation of China (G10502054) and the Knowledge Innovation Program of the Chinese Academy of Sciences (Grant No.KJCX2-YW-L08).

\section{REFERENCES}

[1] Linden, P. F., Lane-Serff, G. F., Smeed, D. A., "Emptying filling spaces: the fluid mechanics of natural ventilation," J. Fluid Mech. 212, 300-35 (1990).

[2] Hacker, J., Linden, P. F., Dalziel, S. B., "Mixing in lock-release gravity currents," Dyn. Atmos. Oceans 24, 183-95 (1996).

[3] Lindken, R., Merzkirch, W., "A novel PIV technique for measurements in multiphase flows and its application to two-phase bubbly flows," Exp Fluids 33, 814-825 (2002).

[4] Nogueira, S., Sousa, R. G., Pinto, A. M. F. R., Riethmuller, M. L., Campos, J. B. L. M., "Simultaneous PIV and pulsed shadow technique in slug flow: a solution for optical problems," Exp Fluids 35, 598-609 (2003).

[5] Carpintero-Rogero, E., Kröss, B., Sattelmayer, T., "Simultaneous HS-PIV and shadowgraph measurements of gasliquid flows in a horizontal pipe," 13th Int Symp on Applications of Laser Techniques to Fluid Mechanics, Lisbon, Portugal, 26-29 June (2006).

[6] Sakakibara, J., Adrian, R. J., "Whole field measurement of temperature in water using two-color laser induced fluorescence," Experiments in Fluids, 26, 7-15 (1999).

[7] Crimaldi, J. P., "Planar laser induced fluorescence in aqueous flows," Experiments in Fluids, 44, 851-863 (2008).

[8] Linden, P. F., "The fluid mechanics of natural ventilation," Annu. Rev. Fluid Mech., 31, 201-238 (1999). 